\title{
MEASUREMENTS ON PLANETARY NEBULAE
}

\author{
D. K. MILNE \\ Division of Radiophysics, CSIRO, Sydney, Australia \\ and \\ L. H. ALLER \\ University of California, Los Angeles, Calif., U.S.A.
}

\begin{abstract}
Parkes $64 \mathrm{~m}$ telescope. These nebulae were chosen to include most of those for which $\mathbf{H} \beta$ flux densities are available. Radio positions and flux densities (generally with probable errors of only a few millijanskies) obtained from the survey must represent the most reliable and homogeneous radio data currently available for the class of object. The data have been used to obtain distances and optical extinctions for these nebulae.
\end{abstract}

\section{Introduction}

One hundred sixty-five planetary nebulae south of $+27^{\circ}$ declination have been observed with the Parkes $64 \mathrm{~m}$ telescope. These nebulae were chosen to include most of those for which $\mathrm{H} \beta$ flux densities are available. Radio positions and flux densities (generally with probable errors of only a few $\mathrm{mJy}$ ) obtained from the survey must represent the most reliable and homogeneous radio data currently available for the class of object. The data have been used to obtain distances and optical extinctions for these nebulae.

\section{Observations}

At $5000 \mathrm{MHz}$ the beamwidth of the Parkes telescope is $4.5^{\prime}$, and with the cryogenic parametric receiver a system noise temperature of $\sim 80 \mathrm{~K}$ was obtained over a 500 $\mathrm{MHz}$ band.

The telescope was scanned in right ascension and declination over the optical position of each nebula many times and the intensity averaged over each arcminute and then integrated over all of the scans. The number of scans varied from six in each coordinate for the stronger sources to 90 for the very weakest. After 20 scans the peak to peak noise level was typically $0.015 \mathrm{Jy}$. In Figure 1 we show 10 integrated scans in each coordinate through the nebula M1-46, for which we obtain a flux density of $0.095 \pm 0.015 \mathrm{Jy}$. The confusion which limited us at $2700 \mathrm{MHz}$ (Aller and Milne, 1972) is apparent in the declination scan but is not a serious limitation here.

\section{Results}

The flux densities obtained here are of much greater accuracy than those from any previous survey. More important, these results are for a much greater sample than previously obtained at this level of accuracy and should tell us something of the interstellar absorption. So firstly, we derived a radio distance scale. This follows from 


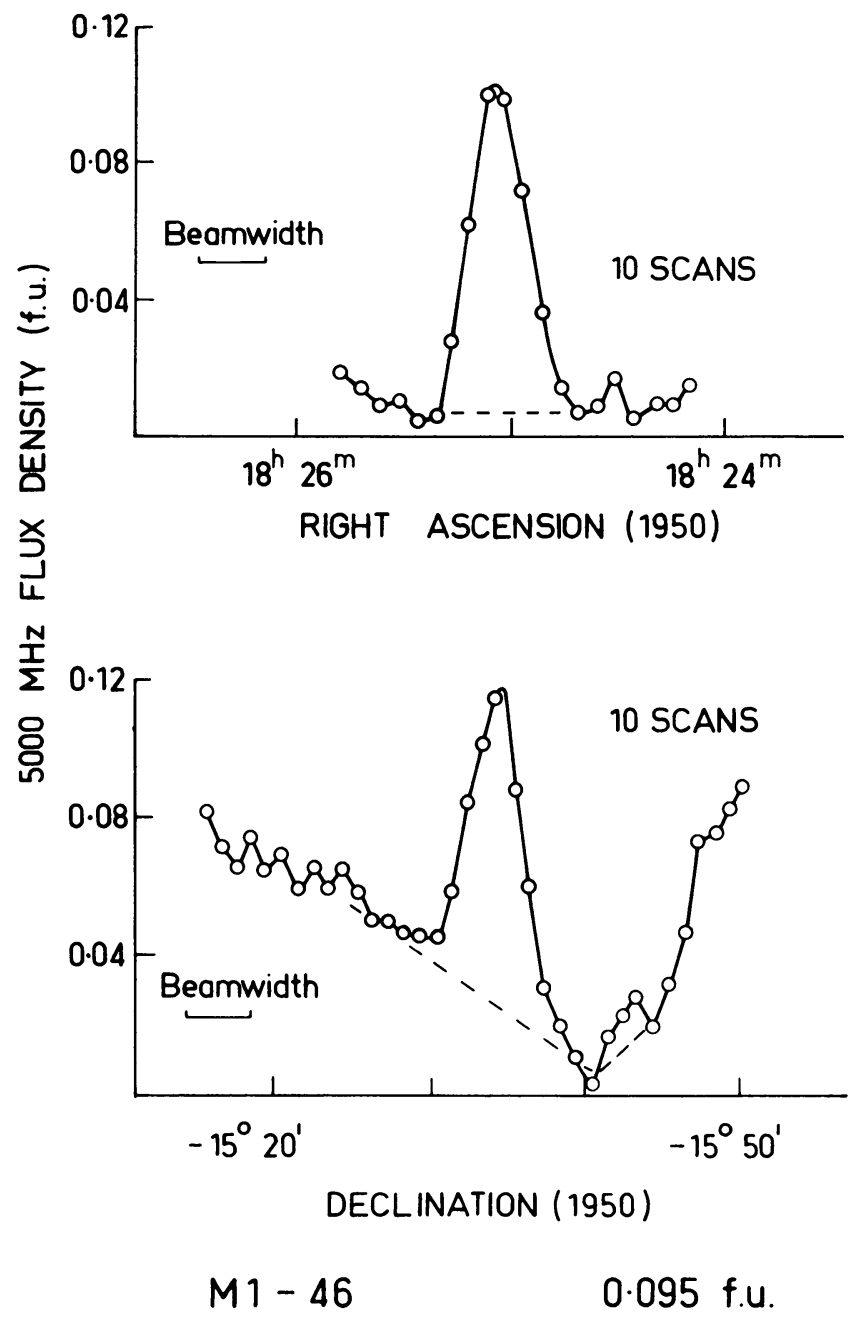

Fig. 1. Averages of 10 scans through the optical position of the moderately weak planetary nebula M1-46 $(0.095 \mathrm{Jy})$ The base levels chosen for each set of scans are shown. The zero point on the flux density scale is arbitrary.

the method of Minkowski and Aller (1954) and Shklovskii (1956) - the constant mass method - in which it is assumed that the same fixed mass of gas is ejected to form the shell in all of the nebulae. One then calculates the free-free radio emission from this gas, arriving at the formula for the distance in parsecs,

$$
d=6180 \theta^{-3 / 5} S^{-1 / 5},
$$

where $\theta$ is the angular radius of the nebula in arcseconds and $S$ is the optically thin radio flux density in janskies. In this derivation a nebular mass of $0.16 M_{\odot}$ and an electron temperature of $10^{4} \mathrm{~K}$ were assumed. 


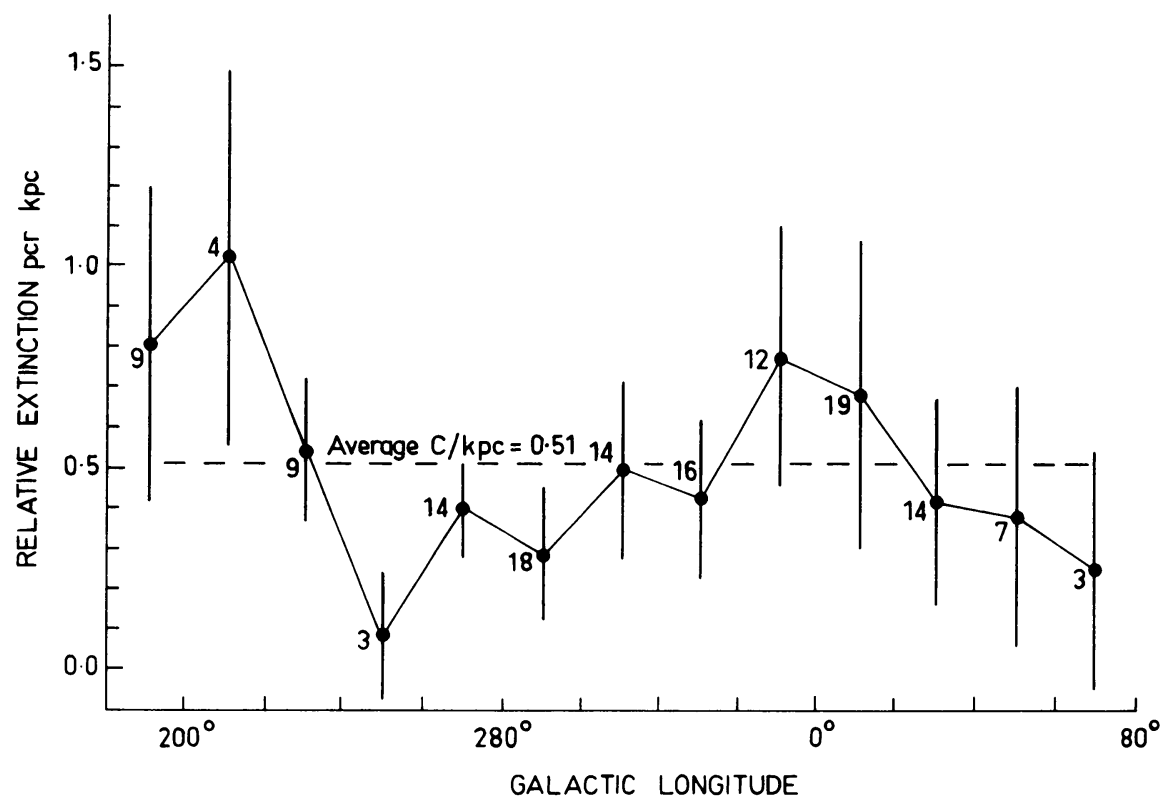

Fig. 2. The extinction per kiloparsec averaged over each 20 deg of galactic longitude. This diagram is based on a galactic disk model $300 \mathrm{pc}$ thick with no absorption outside this disk. The vertical bars represent the error in the average absorption at each $20^{\circ}$ point, the numbers of nebulae averaged in each $20^{\circ}$ sample are shown. The average extinction per $\mathrm{kpc}$ is indicated.

NGC 7293

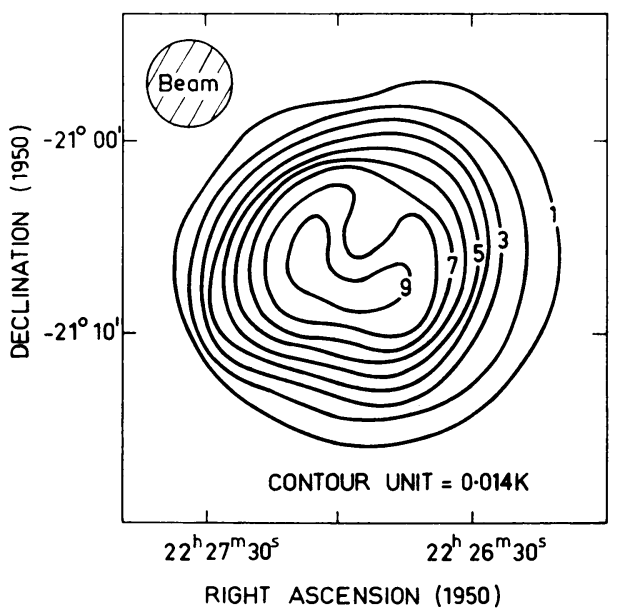

(a) $5 \mathrm{GHz}$ RADIO

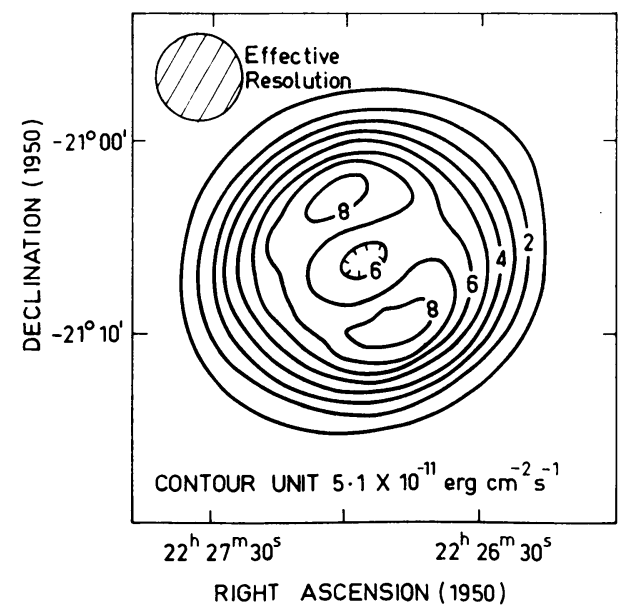

(b) $H \alpha+[N I]$

Fig. 3. (a) $5000 \mathrm{MHz}$ isotherms of the radio emission from NGC 7293. The contour unit is $0.014 \mathrm{~K}$ full beam brightness temperature. (b) $\mathrm{H} \alpha+[\mathrm{N} \mathrm{II}]$ isophotes of NGC 7293 (Gurzadyan 1963) smoothed to the same resolution as the $5000 \mathrm{MHz}$ contours in Figure $3 \mathrm{a}$. The contour unit is $5.1 \times 10^{-11} \mathrm{erg} \mathrm{cm}^{-2} \mathrm{~s}^{-1}$. 
Extinction coefficients were calculated from comparison with the $H \beta$ flux densities. The extinction coefficients obtained here show none of the irregularities seen in previous surveys; at least they are, with two exceptions, all positive within the errors.

Finally, since the distances obtained from Equation (1) are independent of absorption we have calculated values for the mean absorption per kiloparsec. For this we adopted, after some consideration, a uniformly absorbing galactic disk $300 \mathrm{pc}$ thick with no absorption outside this disk. In Figure 2 we show the mean absorption per $\mathrm{kpc}$ averaged each $20^{\circ}$ along the galactic plane. Significant increases in the mean absorption are seen in the direction of Orion and the galactic centre. The average extinction coefficient per $\mathrm{kpc}$ is $0.15 \pm 0.3$ in $\log F(\mathrm{H} \beta)$, i.e., $1.28 \pm 0.75$ mag of absorption per kpc.

There are suggestions (Mottmann, 1972) that the nebular masses increase with time and that a mass of $0.16 M_{\odot}$ is typical of an older nebula, in which case our distances would be upper limits, yielding lower limits on the mean extinction per kpc. How-

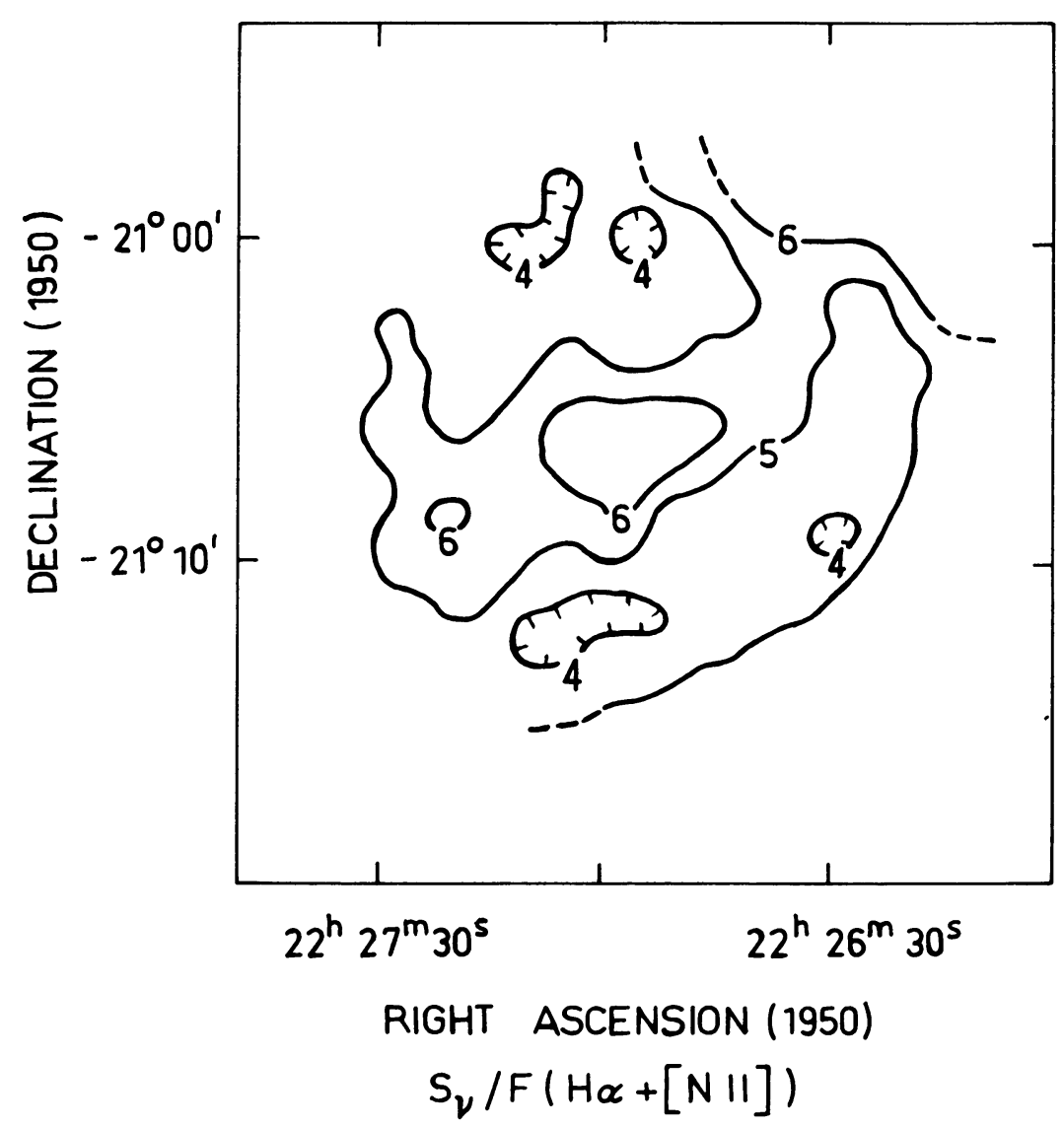

Fig. 4. Contours of the ratio of the radio brightness to the $\mathrm{H} \alpha+[\mathrm{N}$ II $]$ intensity over the nebula NGC 7293. The contour unit is $10^{+8} \mathrm{Jy} / \mathrm{erg} \mathrm{cm}^{-2} \mathrm{~s}^{-1}$, and the effective resolution is $4.4^{\prime}$. 
ever one should bear in mind that many of these objects are more than $150 \mathrm{pc}$ from the galactic plane, so that only part of the distance obtained here is in the absorbing layer.

Only one of the nebulae in this study was resolved in our $4.5^{\prime}$ beam. This nebulae, NGC 7293 (the Helix nebula), was mapped with integrated scans at several declinations. The radio contours in Figure 3 a show the dipole structure of this object. In Figure 3b we have convolved Gurzadyan's (1963) $\mathrm{H} \alpha(+[\mathrm{NII}])$ isophotes to the same resolution as the radio contours. The contour unit in this diagram is $5.1 \times 10^{-11} \mathrm{erg}$ $\mathrm{cm}^{-2} \mathrm{~s}^{-1}$ and was obtained from Metik and Gershberg's (1964) value for the integrated $\mathrm{H} \alpha+[\mathrm{N}$ II $]$ flux density for NGC 7293.

The main difference between Figures $3 a$ and $3 b$ is in the greater angular depth of the indentation in the NW side of the optical features: an absence of optical emission where there is a maximum in the radio contours. This is shown quantitatively in Figure 4, where we have drawn contours of the ratio radio flux density/(H $\alpha+[\mathrm{N}$ II $])$ flux density. Changes in this ratio could be explained either by increased absorption or an increase in the electron temperature over the region of high $S_{v} / F(\mathrm{H} \alpha)$. Assuming that about $20 \%$ of the optical emission near $\mathrm{H} \alpha$ is in the [N II] lines and adopting an electron temperature of $10^{4} \mathrm{~K}$, a value of $4.4 \times 10^{+8}$ in Figure 4 would imply an extinction coefficient of zero. Further the range in $S_{v} / F(\mathrm{H} \alpha)$ in Figure 4 would total a range of $\sim 0.25$ in extinction coefficient and an average value of 0.02 , close to the value of 0.01 obtained from the comparison of radio and $\mathrm{H} \beta$ flux densities. If these variations were to be satisfied by changes in the electron temperature, then temperature differences of $15000 \mathrm{~K}$ would be necessary.

Structure in the interstellar extinction with this angular scale $\left(\sim 10^{\prime}\right)$ is not unexpected. However, Terzian, in this symposium, has shown from his fine synthesis maps of much smaller planetary nebulae that absorption on the angular scale of the smaller objects exists, implying that this absorption is associated with the nebula. If this is so then the mean interstellar absorption would be less than the values that we derive.

We are continuing these observations to include fainter objects and to include those for which optical fluxes are not yet available.

\section{References}

Aller, L. H. and Milne, D. K.: 1972, Australian J. Phys. 25, 91. Gurzadyan, G. A.: 1963, Soobshch. Byurakansk. Obs. Akad. Nauk Arm. S.S.R. 34, 59.

Metik, L. P. and Gershberg, R. E.: 1964, Izv. Krymsk. Astrofiz. Obs. 31, 112.

Minkowski, R. and Aller, L. H.: 1954, Astrophys. J. 120, 261.

Mottmann, J.: 1972, Thesis, University of California, Los Angeles.

Shklovskii, I. S.: 1956, Astron. Zh. 33, 222.

D. K. Milne

CSIRO Division of Radiophysics,

P.O. Box 76 ,

Epping, N.S.W. 2121, Australia 
L. H. Aller

Astronomy Department,

University of California,

Los Angeles, Calif. 90024, U.S.A.

\section{DISCUSSION}

$H$. R. Dickel: What is the excitation class or type of the NGC 7293 planetary nebula? Would a variation of $[\mathrm{NII}]$ also be able to explain optical-radio variation, rather than extinction?

Milne: I don't remember. I think that the rough sum suggested that it would take approximately a $15000 \mathrm{~K}$ variation across the nebula to explain it as an [NII] effect. I believe that it is all differential absorption.

Terzian: I think we should be careful and not overinterpret the interstellar extinction data in deriving 'accurate' distances. Since recent work shows that many planetary nebulae are strong infrared sources, part of the extinction (absorption) may be directly associated with the nebulae.

Milne: I think that the presence of differential absorption on the scale of NGC 7293 and also on the scale of the much smaller objects that you have observed also suggests that this absorption is close to the nebula.

Greenberg: Terzian's comment on the existence of dust in the planetary nebula affecting the extinction raises the important point that one should carefully distinguish between extinction and absorption. Even if there were dust in the nebula it would show up quite differently than if it were somewhere along the line of sight. Roughly speaking, it would tend to exhibit its extinction only as absorption (without scattering), whereas in interstellar space the extinction measures the sum of absorption and scattering. I have suggested this effect in Mem. Soc. Roy. Sci. Liège (1972), p. 197. 